\title{
On a physical model for the formation of the neutron source for s-processing
}

\author{
M. Busso (1), S*. Palmerini (2), E. Maiorca (1), O. Trippella (1), L. Magrini (3), S. \\ Randich (3) \\ (1)Dipartimento di Fisica, Università di Perugia and INFN, Perugia, Italy \\ e-mail: maurizio.busso@fisica.unipg.it; \\ (2) Departamento de Física Teórica y del Cosmsos, Universidad de Granada, Granada, Spain; \\ (3) INAF, Osservatorio Astrofisico di Arcetri, Firenze, Italy
}

The present scenario of the s process in AGB stars emphasizes the role of the neutrons released from the ${ }^{13} \mathrm{C}(\alpha, n){ }^{16} \mathrm{O}$ reaction. This in its turn derives from a local production of ${ }^{13} \mathrm{C}$ in $\mathrm{He}-$ rich layers, after a penetration of protons from the envelope, whose mechanisms have been so far elusive. We speculate that magnetic buoyancy might be at the origin of the mixing process and present a simple model according to which the mixing (hence the extension of the ${ }^{13} \mathrm{C}$-rich layer) should involve most of the He-rich buffer above the $\mathrm{CO}$ core, i.e. a zone much larger than previously envisaged. This implies a large production of $s$-elements in the Galaxy, as is indeed suggested by recent observations in open clusters

12th Symposium on Nuclei in the Cosmos

August 5 - 122012

Cairns, Australia

\footnotetext{
* Speaker.
} 


\section{Extended mixing and magnetic buoyancy in red giants}

The synthesis of nuclei belonging to the main s-process component in AGB stars is known to require the activation of the ${ }^{13} \mathrm{C}(\alpha, n){ }^{16} \mathrm{O}$ reaction for the production of most of the neutrons needed $[1,2]$. Only a marginal second neutron flux, at higher neutron density, derives in the thermal pulses by the reaction ${ }^{22} \mathrm{Ne}(\alpha, \mathrm{n}){ }^{25} \mathrm{Mg}$; see [3] for a review. The formation of the ${ }^{13} \mathrm{C}$ reservoir (or pocket) must descend from the mixing of a limited amount of protons from the envelope into the He-rich zones; in the absence of reliable models for partial mixing in stars, its occurrence has long been the object of free parameterizations. A more quantitative approach for producing ${ }^{13} \mathrm{C}$ in the radiative

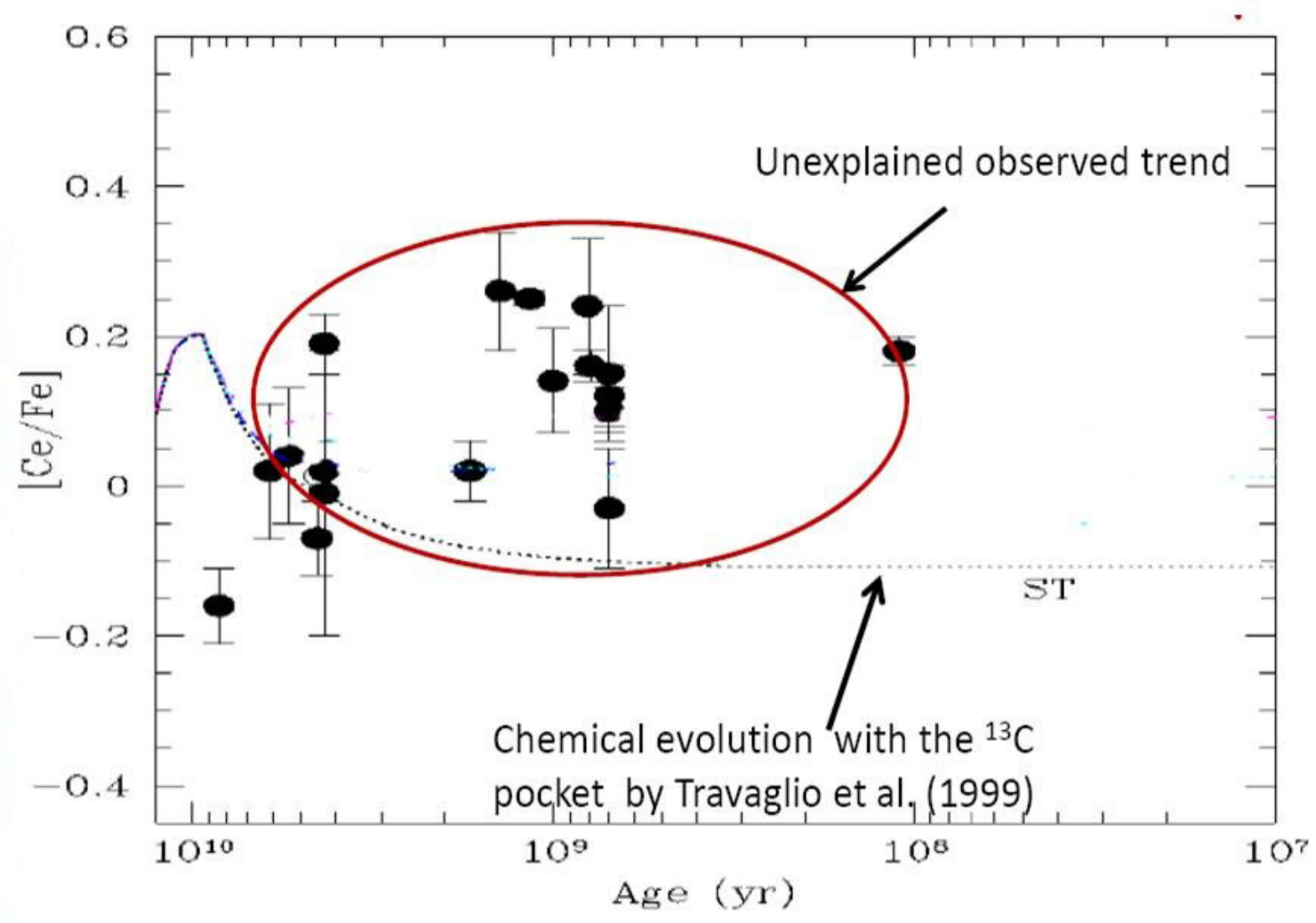

Figure 1: A comparison between recent observations of $\mathrm{Ce}$ in open clusters from [7] and chemical evolution models for the same element computed with the asssumptions made by [5]. Most young open clusters show abundances considerably higher than the models can explain

He-rich regions of an AGB star was then introduced by [4]; in their treatment, it is the convective envelope border that is assumed, more physically than in models using a bare Schwarzschild criterion, to be connected to the underlying radiative zone in a smooth way, with convective velocities that do not drop abruptly to zero, but decrease exponentially. The region of this velocity decrease below the envelope, at the moment when an AGB star experiences the so-called third dredge-up (TDU), naturally produces a partially-mixed region where a few protons remain trapped in He-rich 
layers. While the details of this process are different from older parameterized model by [1, 2], the bulk results are on average similar, even if the theoretical spread in the s-process indexes found by [4] is definitely thinner. The layers involved in the partial mixing in the quoted works are always rather shallow, being typically of the order of $(0.5-1) \times 10^{-3} M_{\odot}$.

Very recently, new observations of neutron-rich elements in young stellar clusters forced us to consider an upgrade of our ideas on the formation of the ${ }^{13} \mathrm{C}$ pocket. The scheme of galactic chemical evolution for a typical neutron-rich element dominated by the s-process, represented as a function of the age of the stellar systems, is shown in Figure 1, taken from [5]. It adopts the scenario for slow neutron captures prevailing so far, where the neutrons are produced mainly by stars of masses larger than $1.5 M_{\odot}$, similar to the typical AGB stars we see today in the solar neighborhoods. They are roughly suitable to explain s-elements in the past stellar generations and their correlation with dynamics [6], and can also address the interplay of the s-process and of the rprocess components in the Sun. However, it is clear from Figure 1 that such models underestimate s-process abundances for the most recent galactic ages. This is so also for other $n$-rich elements, from $\mathrm{Y}$ to $\mathrm{Ce}[7,8]$.

Motivated by the above inputs and limits from the classical studies of the s-process we started a project aimed at modeling the formation of the ${ }^{13} \mathrm{C}$ pocket on first principles, and basing our assumptions on the observational suggestion that requires a more effective $s$-process nucleosynthesis. This cannot be achieved by models increasing the number of protons mixed without also expanding the mass of enriched layers; otherwise we would get a too high number of protons per unit mass, leading the $\mathrm{CN}$ cycling to its full efficiency and producing a lot of ${ }^{14} \mathrm{~N}$, a strong neutron filter hampering the production of heavy neutron rich nuclei.

We therefore had to play with approaches predicting larger polluted fractions of the He-rich zones. This leads quite naturally to think of phenomena linked to the rotational shear present at the border of the $\mathrm{CO}$ core, where the rotation passes from rigid to differential.

Purely rotational effects have however been shown to yield frustratingly small mixing efficiencies, as noted by $[9,10]$. We therefore revisit here the idea, originally presented by [11], that rotation actually favors mixing indirectly, through the maintenance of magnetic dynamo mechanisms, producing the buoyancy of toroidal magnetic structures.

\section{A Model for magnetic buoyancy forming the ${ }^{13} \mathrm{C}$ pocket}

We assumed steady state conditions (no dependence of the rate at which magnetic structures cross a surface from time). In this case the frequency of their arrival at the envelope base, $r_{0}$ is decreased, with respect to the rate $\dot{N}(r)$ characterizing their production at a generic position $r$, by the number of those structures that get dispersed along the path; but it is also increased by the number of those that are born locally between $r$ and $r+d r$.

The magnetic flux associated with dissipated tubes can be neglected in the calculation as, after averaging over the whole spherical surface, it would simply increase a little, through diffusion processes, the average $B$ value of the plasma outside the main flux tubes.

According to the above scheme, in crossing a layer $d r^{*}$ the rate of buoyant structures is diminished by dissipative phenomena that can be collectively described through an absorption coefficient 
$\alpha^{*}$ and is increased by the number structures born there. Hence:

$$
d \dot{N}=-\dot{N} \alpha^{*} d r^{*}+\frac{\varepsilon^{*}}{r^{* 4}} d r^{*}
$$

with $\varepsilon *=\gamma F_{B}^{2}$ and $F_{B}$ is the magnetic flux, assumed as constant. By substituting $r=r * / r *_{0}$ one has:

$$
d \dot{N}=-\dot{N} \alpha d r+\frac{\varepsilon}{r^{4}} d r
$$

where now $r$ is non-dimensional if $\alpha=\alpha^{*} r^{*}{ }_{0}$ and $\varepsilon=\varepsilon^{*} r^{*}{ }_{0}$.

Equation (2) is linear, but non-homogeneous, of the type $R^{\prime}+\alpha R=f(r)$, where $R=\dot{N}$ is the crossing rate. It is generally solved by the sum of a general solution of the associated homogeneous equation $R^{\prime}+\alpha R=0$ and a particular integral of the whole equation. It is then straightforward to show that:

$$
R=R_{0} e^{-\alpha\left(r-r_{0}\right)}+\varepsilon \alpha^{3} e^{-\alpha r} \int_{\left(\alpha r_{0}\right)}^{(\alpha r)} \frac{e^{\alpha r}}{\left(\alpha r^{4}\right)} d(\alpha r)
$$

The value of $\alpha$ refers to dissipation by phenomena like thermal exchanges (with the thermal diffusivity as a coefficient), viscous processes (with the viscosity as a coefficient), magnetic diffusion (with magnetic diffusivity as a coefficient) etc. All these terms, and $\alpha$ itself, are of the order of 1 to a few $\times 10^{3}[12,13,14]$. In such conditions it can be shown, with some tedious algebra, that the second term of equation (3) can be neglected at the first order.

As a first approximation, therefore, magnetic buoyancy might promote the formation of a partially mixed zone, built "from inside out", carrying material from near the CO core to the envelope, to which a similarly-shaped proton penetration phenomenon would follow for mass conservation. The ensuing profile of the proton-rich pocket is roughly exponential (neglecting the second term in equation 3), as in most parameterized approaches. But now it starts to have a physical interpretation, and this last requires that it spreads over most of the He-rich buffer, down to layers where something similar to the solar tachocline is established by the interface between rigid-body and differential rotation. This deep pocket has actually the desired characteristics to justify a larger reservoir of protons (hence of ${ }^{13} \mathrm{C}$ ). the condition of providing a sufficient mixing rate to guarantee an adequate proton transport translates into the condition, already established by [11], that magnetic fields are of the order of $10^{3}$ to $10^{4} \mathrm{G}$ at the envelope base, corresponding to more than $10^{6}$ $\mathrm{G}$ in the deepest layers mixed.

In the next section we shall compare the nucleosynthesis it provides with observations of $s$ elements in the recent Galactic history.

\section{3. $s$-Processing with a Magnetically-driven ${ }^{13} \mathrm{C}$-Pocket}

In order to verify the adequacy of magnetic buoyancy in contributing to the formation of the neutron source ${ }^{13} \mathrm{C}$ for $s$-processing, we have assumed that in low mass stars $\left(M \lesssim 1.5 M_{\odot}\right)$ the ${ }^{13} \mathrm{C}$-pocket has the form of the first term in equation (3), with a value of $r_{0}$ chosen so that most of the mass of the He-rich layers below the TDU extension $\left(4\right.$ to $\left.5 \times 10^{-3} M_{\odot}\right)$ is involved in the proton mixing, hence in the subsequent formation of ${ }^{13} \mathrm{C}$ and in the activation of the reaction ${ }^{13} \mathrm{C}(\alpha, \mathrm{n}){ }^{16} \mathrm{O}$ (see Figure 2). 


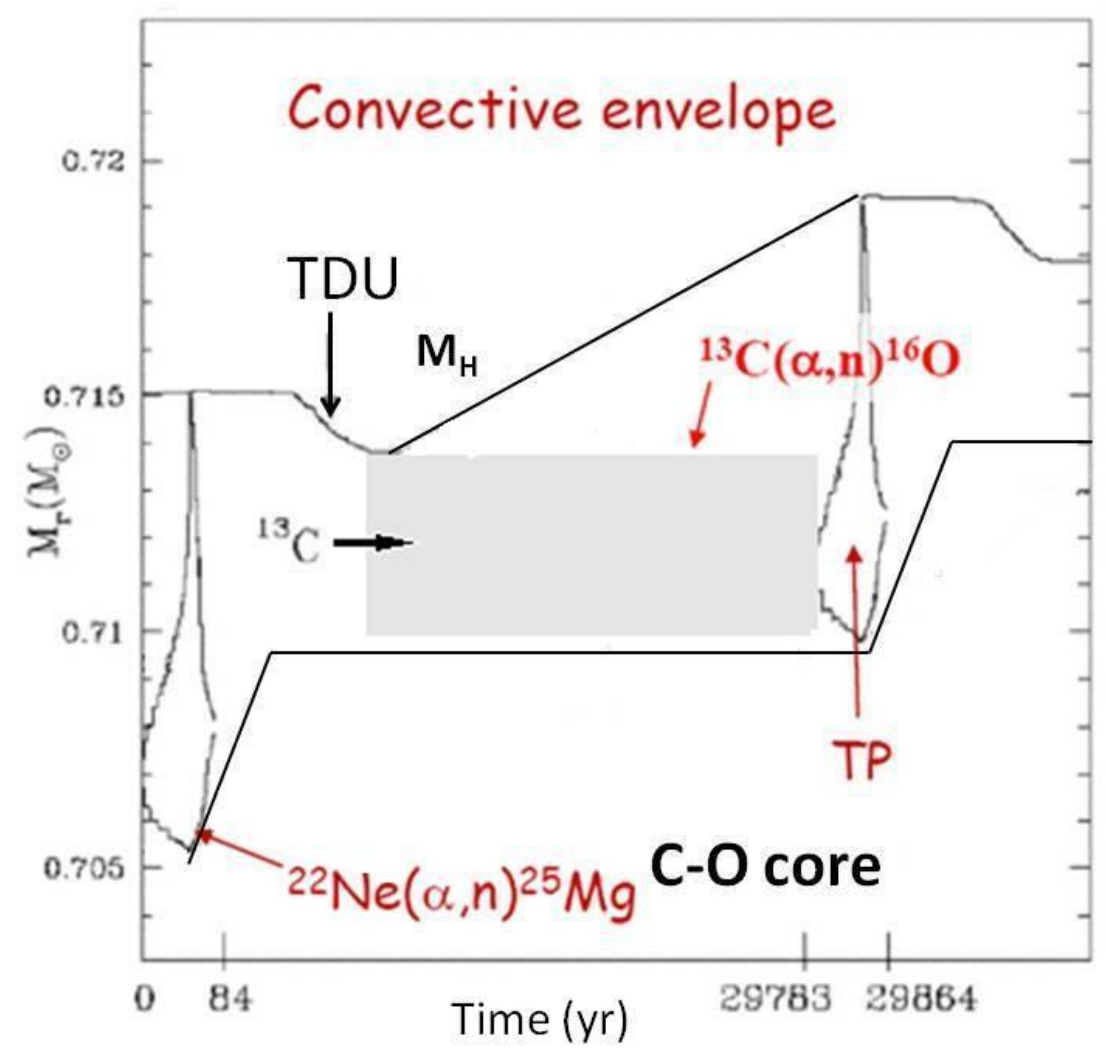

Figure 2: A scheme representing an interpulse stage in an AGB star of low mass, indicating in gray the large area spanned by magnetic buoyancy (hence proton penetration) for the model discussed in the text.

Hence, in this first example, the parameter $\alpha$ of the exponential is fixed so that the abundance by mass of the mixed protons, in layers deeper than the above mentioned mass, becomes sufficiently small $\left(\lesssim 10^{-7}\right)$ to yield negligible ${ }^{13} \mathrm{C}$ and hence negligible neutron generation. The ensuing ${ }^{13} \mathrm{C}$ pocket, after $\mathrm{H}$ re-ignition and $\mathrm{CN}$ burning of the protons thus trapped into the He-rich layers is shown in figure 3.

The scheme illustrated in the Figure is adopted for all masses in the range $1.3-1.5 \mathrm{M}$. , maintaining above this limit the same assumptions for s-processing as in [5], following the idea early presented by [7] that the reproduction of the recent enhancement of $s$-elements in the Galaxy be due primarily to the late contribution of long-living, low mass stars.

Figure 4 (shaded areas) shows the resulting trend of the Galactic chemical evolution for four typical mainly-s elements, namely $\mathrm{Y}, \mathrm{Zr}$, La and $\mathrm{Ce}$. The shaded range is obtained by varying the parameter $\alpha$ of the exponential in equation (1), so that the ${ }^{13} \mathrm{C}$ pocket has an extension bracketed by $4 \times 10^{-3}$ and $5 \times 10^{-3} M_{\odot}$.

As is clear from the plot, the recent increase of $s$-element abundances is well reproduced using ${ }^{13} \mathrm{C}$ pockets of the mentioned extensions in low mass stars; the results obtained with our simple scheme for a magnetically-driven pocket are actually undistinguishable from those by [7], where the pocket for the same stellar masses was chosen ad-hoc. 


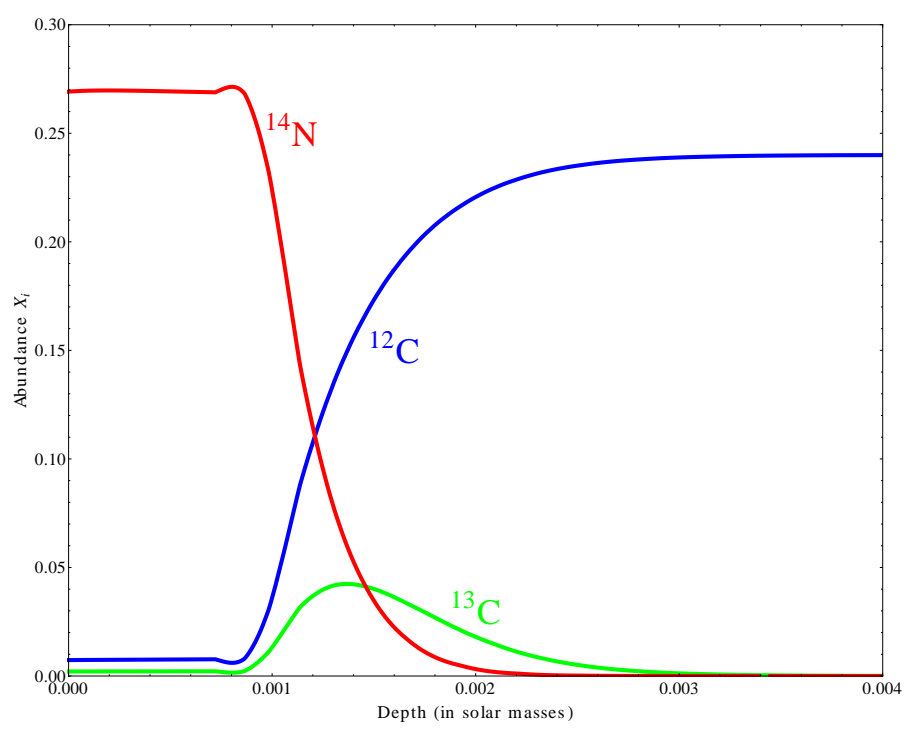

Figure 3: The distribution of ${ }^{13} \mathrm{C}$ and ${ }^{14} \mathrm{~N}$ in the pocket after $\mathrm{H}$ reignition.

Although our approach is still preliminary (in particular the parameter of the exponential function has to be chosen adequately to allow the expression of equation (1) to span most of the He-rich intershell region, in the lack of knowledge of where precisely magnetic flux tubes can detach, near the rotational shear) the fact that a reasonable physical interpretation exists remains important.
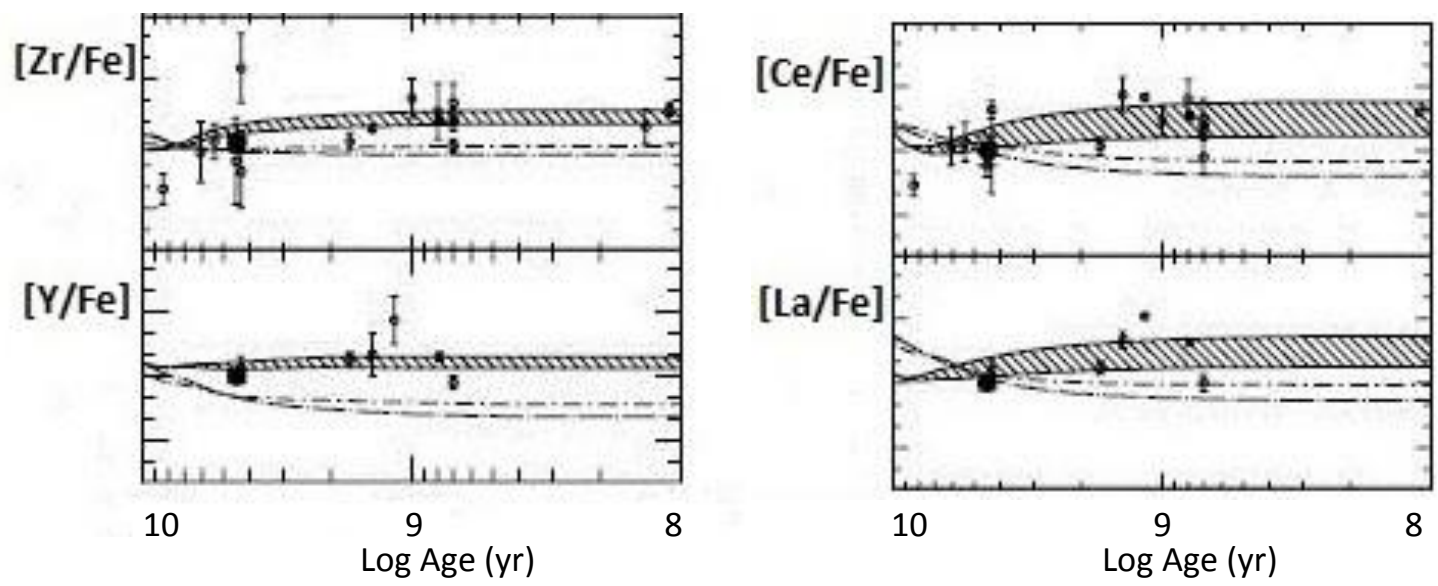

Figure 4: The Chemical evolution of the Galaxy in s-elements, as reproduced with the new assumptions for the ${ }^{13} \mathrm{C}$ pocket for masses below $1.5 M_{\odot}$ (continuous lines bracketing the shaded areas) versus older models, with a pocket extending for $10^{-3} M_{\odot}$, either including (dash-dotted lines) or excluding (dashed lines) the stars below $1.5 M_{\odot}$. 
One relevant point is that our idea is based on a necessary property of the rotating stellar plasma (that of embedding toroidal magnetic flux tubes, maintained by dynamo effects); we believe this model now deserves to be explored in depth, in order to verify under which conditions it can yield a non-parametric solution to the formation of the main neutron source for $s$-processing

\section{References}

[1] C. Arlandini, F. Kaeppeler, K. Wisshak, et al., ApJ 525 (1999) 886

[2] R. Gallino, C. Arlandini, M. Busso, M. Lugaro et al., ApJ 497 (1998) 388

[3] M. Busso, R. Gallino, \& G.J. Wasserburg, ARAA 37 (1999) 239

[4] S. Cristallo, O. Straniero, R. Gallino et al., ApJ 696 (2009) 797

[5] C. Travaglio, D. Galli, R. Gallino, \& M. Busso, ApJ 521 (1999) 691

[6] C. M. Raiteri, M. Villata, M., R. Gallino, M. Busso, A. Cravanzola, ApJ 518 (1999) L91

[7] E. Maiorca, L. Magrini, M. Busso, S. Randich, S. Palmerini, \& O. Trippella, ApJ 747 (2012) 53

[8] D. Yong, B.W. Carney, \& E.D. Friel, 2012, AJ 144, 95

[9] A. Palcios, C. Charbonnel, S. Talon \& L. Siess, A\&A 453 (2006) 261

[10] C. Charbonnel \& N. Lagarde, A\&A 522 (2010) 10

[11] M. Busso, G.J. Wasserburg, K.M. Nollett \& A. Calandra, ApJ 671 (2007) 802

[12] E.T. Vishniac, ApJ 446 (1995) 724

[13] E.T. vishniac, ApJ 451 (1995) 816

[14] G. Pascoli, \& L. Lahoche, PASP 122 (2010) 1334 